\title{
Effect of Hollow Blades on Flow Improvement in Turbines and Large Scale Fans
}

\author{
Ali Najafzadeh ${ }^{1 *}$, Ali Zare², Behzad Shahizare³, S. Meysam Kosari Esfahan ${ }^{4}$, \\ Iman Asadi ${ }^{5}$ and Naeim Ezzat Ahmadi ${ }^{6}$ \\ 1,2,3,5,6 Department of Mechanical Engineering, Universiti Tenaga Nasional, Kajang, Malaysia; \\ Alinajafzade1@gmail.com¹,Ali_z4688@yahoo.com²,Shahizare@gmail.com³, Iman_asadi64@yahoo.com5, \\ Ahmadi_naeim@yahoo.com ${ }^{6}$ \\ ${ }^{4}$ Department of Engineering, Islamic Azad University, Najafabad Branch, Iran; M.kosari8520@yahoo.com
}

\begin{abstract}
In this paper different computational fluid dynamics (CFD) methods have been defined and modern methods are developed and compared with conventional ones. Numerical methods are used to calculate volume flow rates of turbines and axial flow fans. Validation of results with experimental data is improved significantly by using hollow blades and this will result to increased efficiency of turbines and large scale fans.
\end{abstract}

Keywords: Computational Fluid Dynamics (CFD), Axial Flow Fans, Volume Flow Rate, Hollow Blade Turbine.

\section{Nomenclature}

Q volume flow rate, CMM

$\mathrm{P}_{\mathrm{s}}$ static pressure, $\mathrm{Pa}$

$\eta_{s}$ efficiency for static pressure

$\omega$ nominal rotation speed, $\mathrm{rpm}$ qv, int volume of internal flow, $\mathrm{m}^{3}$

qv, ext volume of external flow, $\mathrm{m}^{3}$

Op power input, $\mathrm{KW}$

\section{Introduction}

Computational fluid dynamics (CFD) method has provided useful and more effective approach to simulate, design, optimize and analyze flow of fan during 1970s and 1980s [13]. Numerical techniques and turbulence models have been developed highly for applications of fans with inter-blade flows. Simulation for efficiency prediction was also a main issue that was focused upon in 1990s. Turbulence models and CFD techniques are being used for turbo machinery flow analysis and to study the flow phenomena in axial rotors with different skewed blades, then numerical method has been got into the process to validate the results experimentally. A method to design skewed blade was developed base on simulation and theoretical reasoning fan blades was reducing noise generation and improving fan performance during 2000s. An actuator disk model was developed by Meyer and Kröger [14] to simulate velocity field and performance of an axial flow fan. Although a dramatic improvement took place and only $10 \%$ to $20 \%$ deviation was obtained from the experimental results, a discrepancy appeared downstream due to the omission of tangential and radial forces from the blade tips and fluid stream by combination of presented disk models with simplified ones which are in porous type and perform a numerical method to investigate on the effect of performance on aerodynamic behavior of a air-cooled heat exchanger. New results showed aerodynamic behavior

* Corresponding author:

Ali Najafzadeh (Alinajafzade1@gmail.com) 
to be affected by volume flow rate and root stagger angle of blades during this operation. Afterward, leakage effects of the tip clearance on fan performance, flow structure and noise generation have been studied. So optimizing the tip clearance has become another goal that would lead to reduction of noise and escalation of fan performance.

A new approach to study flow field and performance characteristics of an axial flow fan is "downstream flow resistance" (DFR) which has several advantages over conventional method that are popular among fan designers. Firstly, deviation of curves calculated for showing fan performance, from experimental results will be less than $3 \%$ and $1.5 \%$ with static and moving grid schemes respectively, while the maximum deviation obtained conventional method is $26 \%$ with static-grid scheme [13].

A dramatic improvement has been reached by using DFR. Secondly, in DFR method identical results will be shown for both moving and static grids, so usage of static and moving grids to predict fan performance will not be an issue anymore. Thirdly, in conventional method stall occurs if contour of fan blade is not designed appropriately for operations with high and mid back-pressure. Recirculation bubble would appear under this situation around trailing edge of the fan blade and blocks the development of the flow at deep stall. Large recirculation can also take place around the pressure surface which obstructs flow passage and causes reduction of flow rate. Axial fans pass fluid (gas or air) through an approximately axial direction. Due to their multi-function ability, axial fans have been used mainly in commercial, industrial, residential and institutional applications. So, improving the design of an axial fan would be always an issue to achieve green policy and reduce reverse impact on environment, reduction in noise, and also optimize the design of blades and vanes to get the highest possible performance of an axial fan. Several types of designs like semi-empirical are proposed to enhance the shape of blades and vanes [13].

Different fan characteristics are usually represented by several varieties including total pressure, rotation speed, static pressure, mechanical and static pressure efficiency, motor input, sound level, flow rate, etc. Fan performance has usually been indicated by flow rate and static pressure, so a separate variable termed as performance curve is defined to show the relationship between these two parameters. CFD techniques have been got in process to predict the performance curve and get the optimal performance of the entire fan. In such techniques several boundary conditions will be defined during simulation process like constant flow rate, uniform back pressure and constant inlet pressure. By measuring pressure and velocity distributions using numerical methods, the fan performance will be calculated, regarding the relationship between flow rate and static pressure. The results have accuracy of approximately $80 \%$ by using this approach which is not satisfactory to design an axial fan. So the performance should get improved more by changing several parameters such as moving grid strategy, grid structure and deployment, boundary conditions, turbulence models, etc. By varying mentioned parameters, deviation from empirical results can be reduced which is the primary goal of designing.

Experimental investigation has been performed on the external and internal flow interaction around hollow NACA series airfoil [5]. The trailing edge region is being studied in details and turbulence intensities and velocity has been measured. With the aid of computer visualization, flow structure on the hollow airfoil is being developed. Furthermore, the influence of the internal flow on the flow field of the axial fan was investigated. A reduction of circulation effects has been occurred on the fan hollow blade base on introduction of the internal flow. Characteristic related to aerodynamics of the axial fan is increased to a higher degree of the normalized efficiency and total pressure difference.

A fast method of design is defined for inlet guide vanes which are positioned within two surfaces with conical shapes and constant thickness distributions except the trailing and leading edges regions [10]. The conical inlet guide vane is designed by using quasi three-dimensional calculation method. The empirical results are being compared with pre-designed conditions and with numerical results from three-dimensional viscous and inviscid flow computed with FLUENT code.

\section{Numerical Methods (Computational Procedure)}

Based on experimental conditions, DFR method specifies an entire pressure on exterior surfaces of inlet domain but conventional method defines a back pressure over the duct outlet, directly. The static grid method shows flow surfaces of the stator, shroud and rotor as wall conditions in relation to their reference frames. In opposite, the moving grid method terms the rotor films as the rotating wall boundaries in relation with a reference frame.

Time step should be achieved by estimation of fan speed and is approximately A for moving grid scheme. 
The residuals which are normalized of physical quantities shouldn't be more than B by consideration of convergence visibility and computational accuracy. Furthermore, the convergence criteria for variations of flow rate and back pressure between two adjacent iterations should be less than C. Space modeling of inside the fan is filled with small grids to keep the geometry features of the fan.

\section{Experimental Process to Test Fan Performance}

In this test, the facility is a rectangular chamber which involves nozzles, pressure measuring devices, auxiliary fan, and mesh screen (to settle the flow) according to AMCA standard (ANSI/AMCA 210-99, 1999). A schematic view of the chamber is shown in Figure 1.

Dimensions of the chamber are $0.56 \mathrm{~m} \times 0.56 \mathrm{~m} \times$ $1.76 \mathrm{~m}$ for inner surfaces. Static pressure rise is termed as difference between total pressure at the fan inlet and static pressure at the fan outlet. By measuring pressure drop (across the nozzles) airflow rate will be obtained. In selection of the nozzle(s), Reynolds number is an issue and has to be greater than 12,000, so discharge coefficient of the selected nozzles(s) will be 0.95 (deviation of flow rate for the sonic nozzle from standard flow rate measured by test rig is around $0.8 \%$ in maximum). A probability of $95 \%$ is chosen to be acceptable for uncertainties in experimental quantities which are calculated by statistical analysis of repeated tests. These uncertainties can be divided into two parts: combined and measurement. According to AMCA standard, first part is the result of using various quantities and each quantity is associated with a measurement uncertainty. A combined uncertainty is termed as characteristic uncertainty which is created of measurement one for static pressure versus flow rate. In similar, another combined uncertainty called the uncertainty of static pressure efficiency is obtained from measuring the rate of static pressure efficiency versus flow. Maximum air flow rate $(\mathrm{Q})$, nominal rotation speed $(\omega)$ and maximum pressure rise for the fan used in this test are $0.54 \mathrm{CMM}, 15,000 \mathrm{rpm}$ and $33.4 \mathrm{mmAq}$, respectively. Nominal rotation speed is the rotor's rotation speed which is measured at free delivery. However, this rotation speed varies because no servo system is embedded in the design of motor control of this fan. So, a correlation will be conducted between delivered flow rate and rotation speed.

Figures 2 and 3 are showing the changes of static pressure for measuring fan performance and static pressure efficiency over fan flow rate.

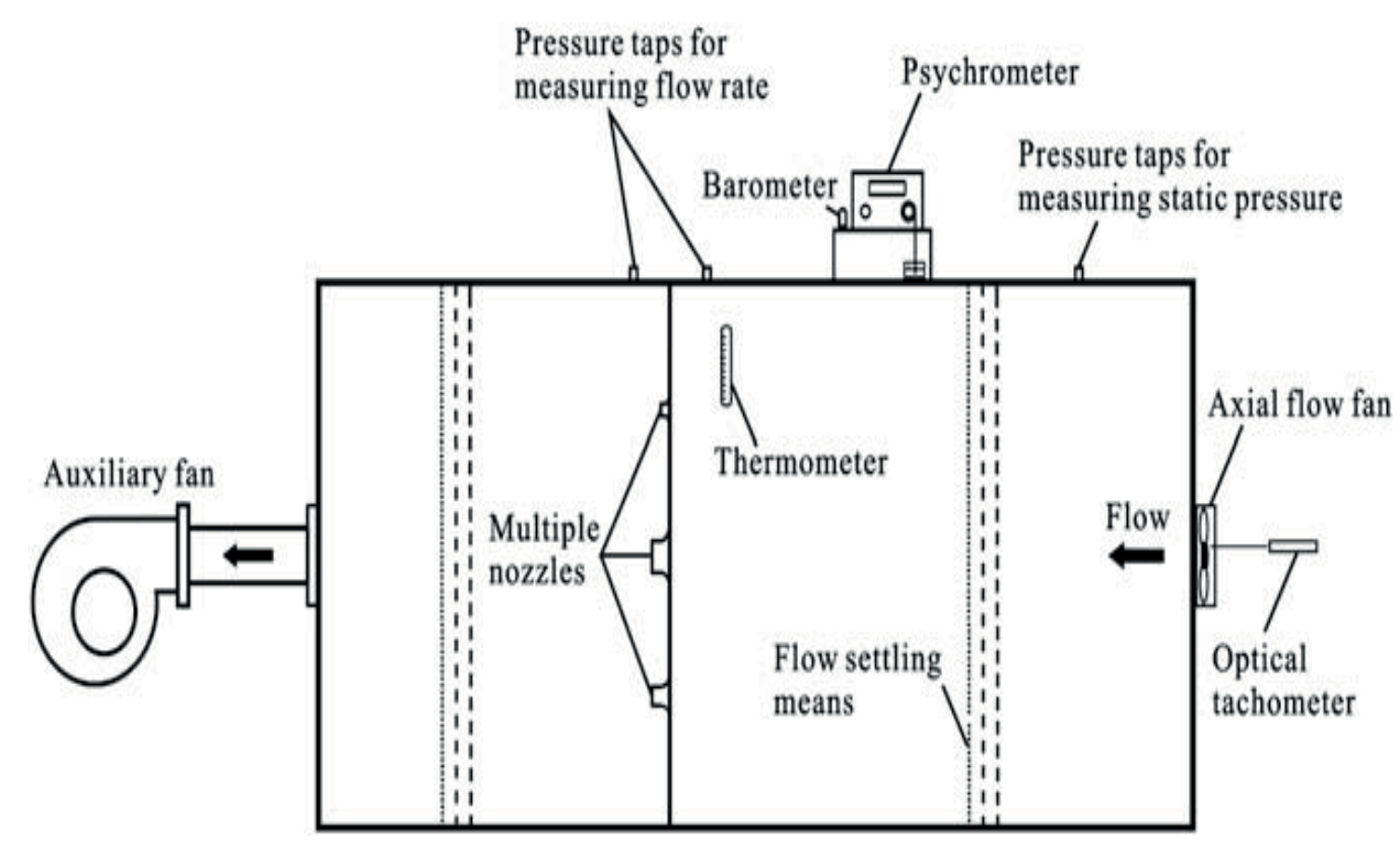

Figure 1. Facility for measurement of fan performance. 


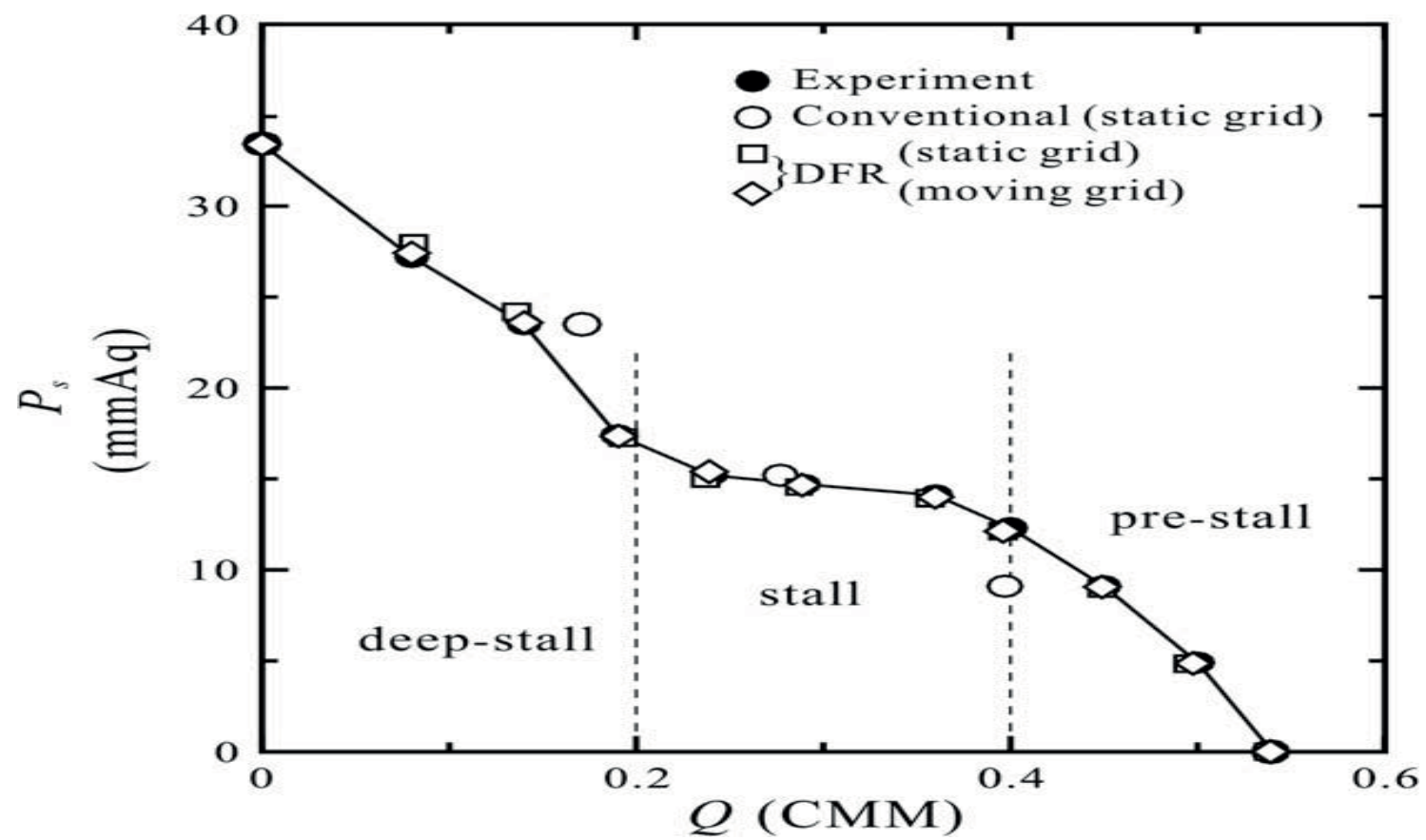

Figure 2. Calculated and measured fan performance curves.

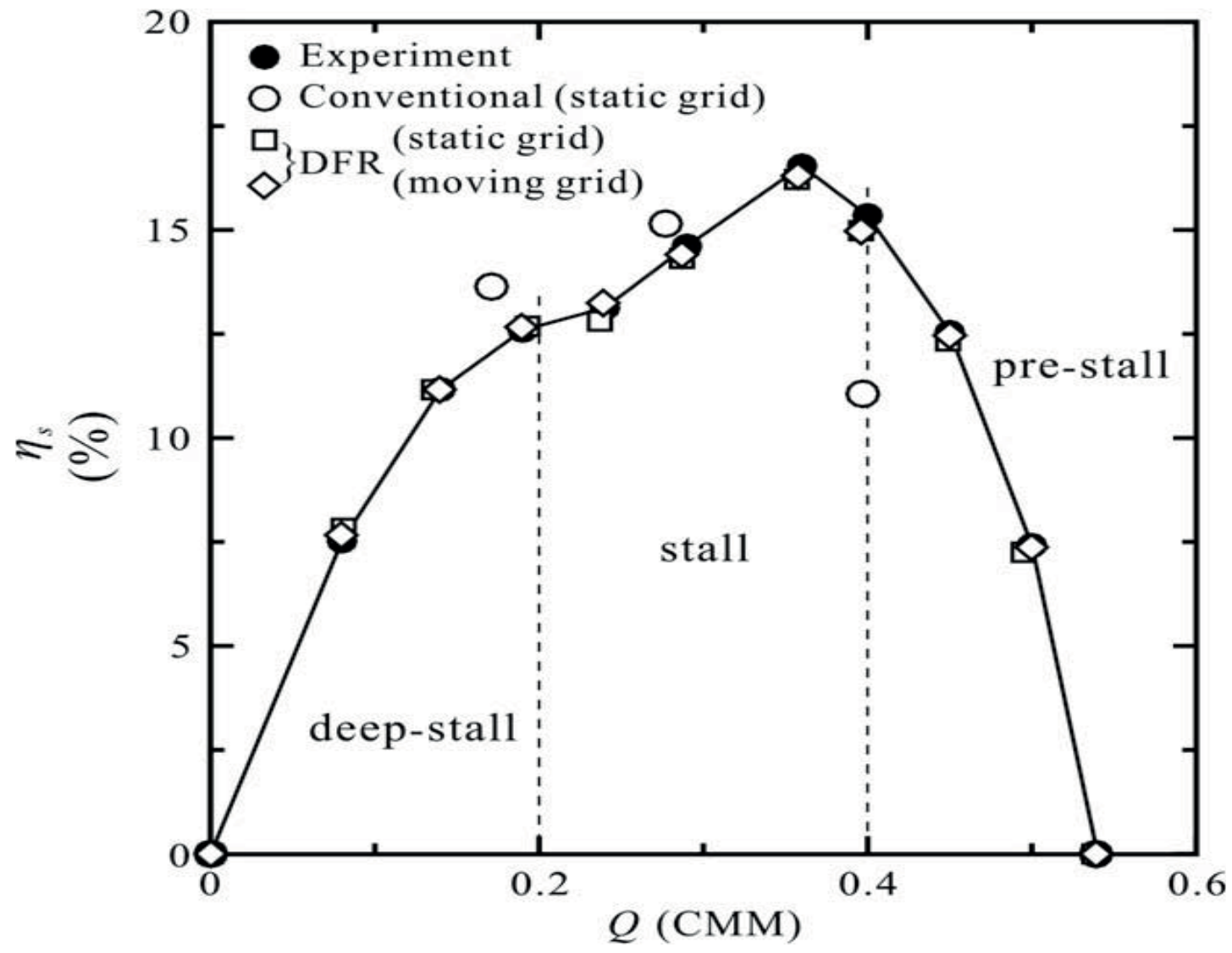

Figure 3. Calculated and measured static pressure efficiency. 
It's obvious that deviation from experimental results is much less by using DFR (especially moving grid). The graphs are divided into three regions: deep-stall, stall and pre-stall. Maximum $\mathrm{Q}$ is derived from pre-stall region which is about $0.54 \mathrm{CMM}$ when $\mathrm{P}_{\mathrm{s}}=0$ (i.e. outlet and inlet pressure are equal to each other). Decreasing rate in pre-stall region is about $88 \mathrm{mmAq} / \mathrm{CMM}$ which is a little larger than about $84 \mathrm{mmAq} / \mathrm{CMM}$ for deep-stall region. Maximum deviation for fan performance measurement from experimental results for conventional method is $26 \%$ at $\mathrm{Q}=0.4 \mathrm{CMM}$, but this digit for static and moving methods is only $3 \%$ and $1.5 \%$, respectively. DFR method can achieve low deviations from experimental results due to the iteratively adjusted back pressure to obtain values which are more realistic during the computational process. The amazing part is that, there is no significant difference between moving-grid and static-grid schemes during fan performance predictions when we employ DFR method.

The maximum efficiency for static pressure is $\eta_{s}=$ $16.54 \%$ which occurs at $\mathrm{Q}=0.36 \mathrm{CMM}$. Maximum deviation for static pressure efficiency using DFR moving-grid, DFR static-grid and conventional methods are 2.5\%, $2.8 \%$ and $28 \%$, respectively. So, using DFR method especially with static-grid scheme for fan performance prediction will shorten the computational time and also increase accuracy.

\section{Experimental and Simulations}

\subsection{Advanced Renormalization Group for Large Scale Motor}

Advanced renormalization group (RNG) has been obtained from instantaneous Navier-Stocks equations and is completely suitable to solve rotating problems [22]. Simple method and second-order upwind is performed to improve the accuracy. Flow behavior is calculated using a multiple reference frame (MRF) modeling. Rotating motion of the shaft, rotor and fans is modeled by MRF function in CFD tool. In all equations except the energy, maximum sum of the normalized residuals will satisfy the convergence.

For large-scale motor, external air enters the air-to-air heat exchanger through guide vanes which are embedded at the outlet of centrifugal fan and internal air is pumped by axial fans and passes through rotor and stator in parallel paths at the same time. Schematic view of the motor is shown in Figure 4.

\subsection{Interaction of Two Flows on the Axial} Fan Hollow Blades

Adverse flow conditions often take place at the tip of the blades and at the hub in axial fans [2]. Recirculation flow occurs at the region of the inlet slot near the fan hub. In this location, the internal flow enters the hollow blade and exits it at the trailing edge as free jet as shown in Figure 5.

Numerical and analytical calculation has been performed and qv, int/qv, ext (The ratio of volume between internal and external flow) is measured which ranges from $1 \%$ to $1.5 \%$ [8]. Internal flow direction being defined by the shape of blade and ejector effect at the trailing edge is obtained according to this defined direction. Furthermore, reduction of flow separation, boundary-layer reduction and reduction of adverse flow conditions at the tip of the hollow blade has been taken place. Flow particles in the boundary-layer do not flow towards the leading edge from the trailing edge. So, flow separation would not occur [12].

Several investigators have explained the concept of reducing blade wake through trailing edge blowing. Schlichting [17] has developed a method to prevent flow separation by means of supplying extra energy into the fluid particles. Design of aircraft wings has been modified by applying suction in the wings by Rhee et al. [16] which resulted to maximum lift. Garg [11] used hollow blades in gas turbine with induced secondary flow circulation in cooling system. Murthy et al. [15] described the system numerically and empirically which has application in chemical industry to mix multi-phase fluids. Bleier [1] presented a $508 \mathrm{~mm}$ diameter vane-axial fan wheel with compressed-air jets. High velocity jet of fan compressed air was produced and ejected through the tip of the blade at trailing edge region. Sutliff et al. [19] and Woodward et al. [21] reduced turbo fan noise with full-span trailing edge blowing with internal flow in radial flow paths. An axial fan with $500 \mathrm{~mm}$ outer diameter and seven hollow blades was made and tested [4]. From the analysis, turbulence intensities and circulation effects on the solution side of the hollow blade were decreased but velocity values presented greater amounts due to the introduction of internal flow.

For two cases of the fan: with and without internal flow, measurements have been performed. Measuring was at equal ambient and operating conditions according to the standard (Standard) which results the overall measuring uncertainty to be $2.8 \%$ of the measured value. The aerodynamics characteristics of the axial fan are showed in Figure 6. 


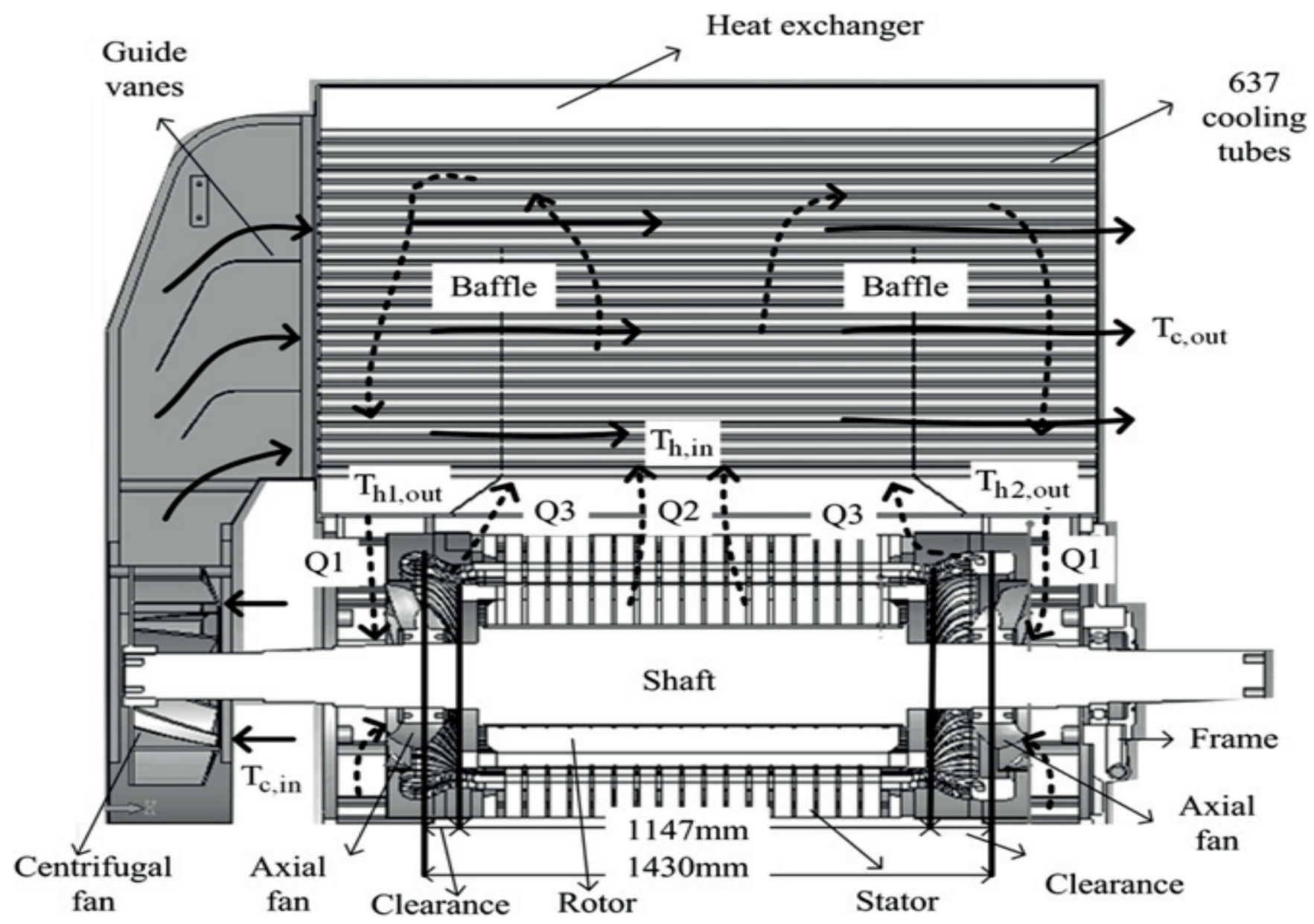

Figure 4. Schematic view of flow paths and components for the motor.

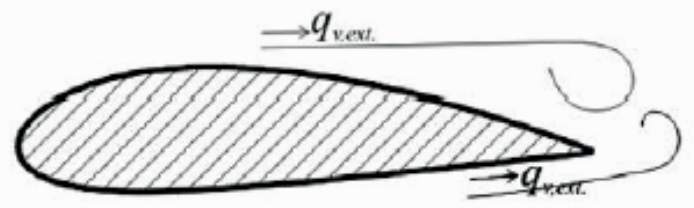

a)

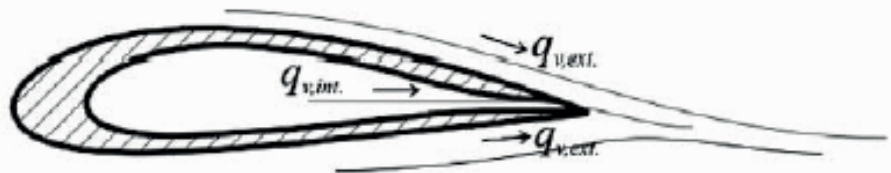

b)

Figure 5. Flow field around the hollow airfoil: a) without internal flow b) with internal flow.

\section{Results and Discussion}

Numerical predictions will be verified by comparing the experimental data with simulated results for original design. In order to enhance the thermal performance of the cooled motor, modified designs of the guide vanes, heat exchanger, axial fans, and clearance among the fan, rotor and stator are also proposed. By increasing the mass flow rate, input power increases but static pressure will be reduced and the air goes through the blade surfaces from the space between the blades.

The largest value for power input (Op) is $7.64 \mathrm{KW}$ which occurs at $4.49 \mathrm{Kg} / \mathrm{s}$ for mass flow rate in centrifugal fan and the lowest amount of Op is $2.31 \mathrm{KW}$ at 1.84 $\mathrm{Kg} / \mathrm{s}$ mass flow rate for the axial fan. Iron core temperatures are empirically measured which are $111^{\circ} \mathrm{C}$ for large pressure drop of the axial fan and small pressure drop for centrifugal fan and are $1^{\circ} \mathrm{C}$ lower than calculated results. 


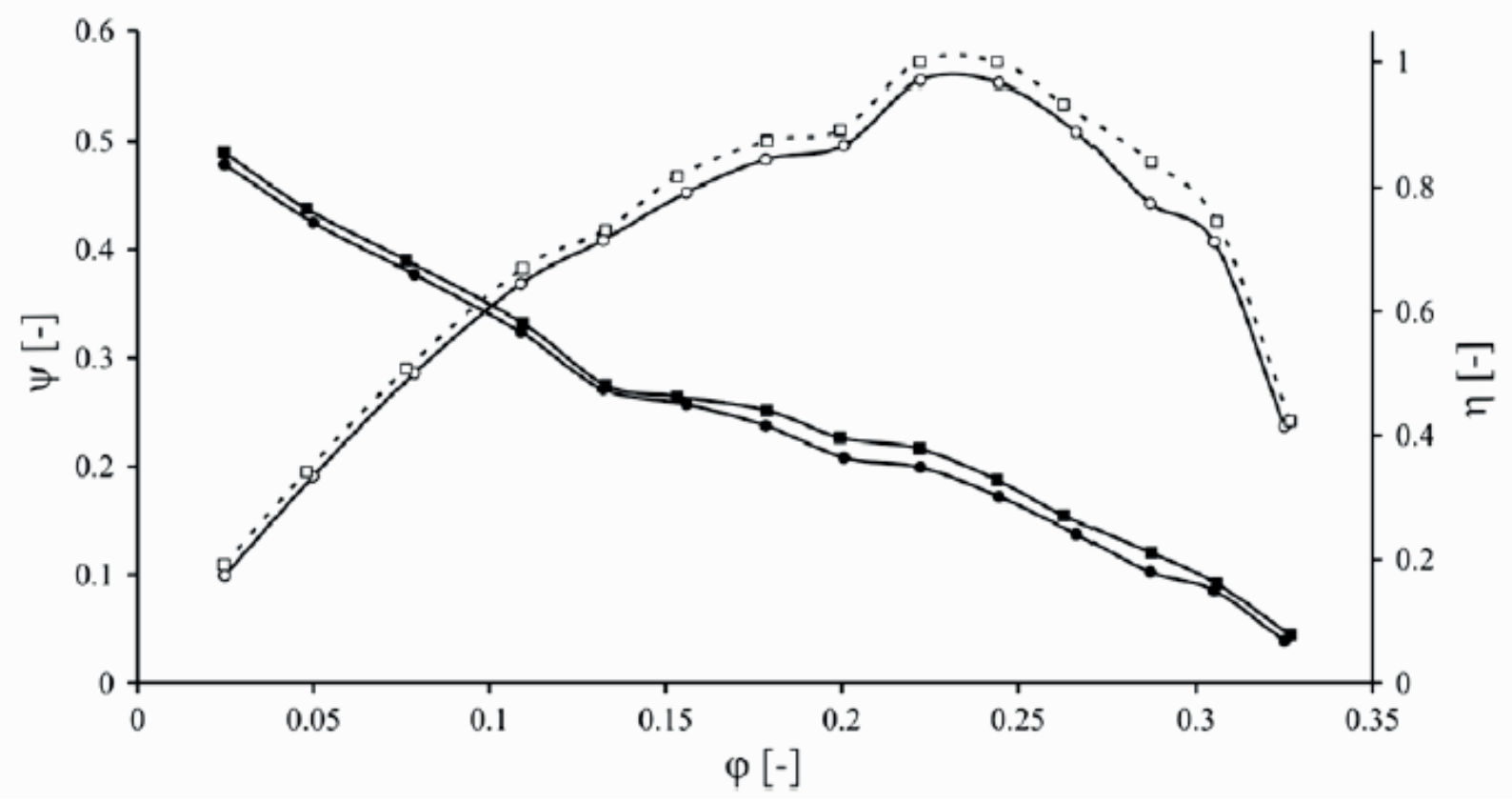

Figure 6. Comparison of aerodynamic characteristic of axial fan at local properties and efficiency for the case with and without internal flow. (-) Total pressure coefficient $\psi$ for the case with internal flow, $(\bullet)$ Total pressure coefficient $\psi$ for the case without internal flow, $(\square)$ efficiency $\eta$ for the case with internal flow, $(\bigcirc)$ efficiency $\eta$ for the case with internal flow.

The calculation of temperatures for copper winding would be $120^{\circ} \mathrm{C}$ and $131^{\circ} \mathrm{C}$ for small pressure drop of the axial fan and large pressure drop of the centrifugal fan, respectively and have approximately $2^{\circ} \mathrm{C}$ and $7{ }^{\circ} \mathrm{C}$ deviation of the experimental results [3].

Temperature distribution on the rotor is more uniform than the stator due to the rotational motion of the rotor. To enhance the performance of the motor, the design parameters of the centrifugal fan are modified including blade height from $168 \mathrm{~mm}$ to $223 \mathrm{~mm}$, inner diameter of fan blade which varies from $400 \mathrm{~mm}$ to $440 \mathrm{~mm}$ and number of blades from 8 to 14 . Highest amount of mass flow rate (MFR) are $1.67 \mathrm{~kg} / \mathrm{s}$ and $1.81 \mathrm{~kg} / \mathrm{s}$ which occur at $400 \mathrm{~mm}$ of inner diameter of fan blade and $223 \mathrm{~mm}$ of the blade height. Flow distribution has negative effect on MFR and when this phenomenon reduces, mass flow rate becomes larger. Also, MFR has the largest value of $1.63 \mathrm{~kg} / \mathrm{s}$ at 14 blades.

Two calculations of total efficiency are performed with and without fan's input power which have the amounts $97 \%$ and $97.5 \%$, respectively. So, these two efficiencies have almost same value and fan's input power doesn't influence the total motor efficiency so much. After measuring MFR for different parameters of the centrifugal fan, it is found that MFR has the maximum value of $1.87 \mathrm{~kg} / \mathrm{s}$ which is $15 \%$ greater than the amount for original design. The number of guide vanes is varied from two to four with one vane positioned vertically and three others at top center of centrifugal fan. After modification, MFR is distributed and has the value of $1.27 \mathrm{~kg} / \mathrm{s}$.

The other main parameter that should be optimized is the distance between the axial fans. In order to increase the efficiency, this length is decreased from $1450 \mathrm{~mm}$ which is the original design to $1360 \mathrm{~mm}$. The axial-flow rate (Q1) is divided into two flow rate: Q2 which passes through the shaft and Q3 flows into clearance. When L is $1400 \mathrm{~mm}, \mathrm{Q} 3$ has the optimum value of $0.194 \mathrm{~m}^{2} / \mathrm{s}$ and the rate of Q2/Q1 is at the greatest value which is $92.1 \%$. So, at the length of $1400 \mathrm{~mm}$, stator and rotor can be effectively cooled by the internal air. At the amounts below $1400 \mathrm{~mm}$, flow resistance in the clearance becomes larger which results in lower cooling capacity for stator and rotor, and at the amounts greater than the optimum value, air leakage increases due to increasing flow channel among the axial fans, rotor and stator. 


\section{Conclusion}

A new numerical method is developed for fan performance prediction which is different from conventional methods. The downstream flow resistance (DFR) method will improve the accuracy of pressure difference prediction between the inlet and exit of the axial fan when compared with conventional methods in which a fix static pressure at the fan exit is specified. Using static-grid and moving-grid DFR method will decrease fan performance curve deviation from $26 \%$ to $3 \%$ and $1.5 \%$, respectively. Static pressure efficiency deviation will also be dramatically reduced from $28 \%$ to $2.8 \%$ and $2.5 \%$ for static-grid and moving-grid, respectively. So, computational time will be decreased and accuracy will be highly increased by performing DFR method instead of conventional ones.

Empirical investigation shows that getting an effective film cooling coverage is very difficult in the front part of endwall surface because of the stagnation region which promotes jets lift off in front part of the endwall surface. So, using fan-shaped holes especially with conical exists are necessary in order to enhance coolant thermal protection capability. Conical holes provide a better improvement in endwall surface protection in comparison with cylindrical ones. This should be get under consideration when more cooling performance is required in industrial applications. Cooling system for large scale motors should get improved due to industrial application requirement of higher reliability and efficiency motors. Improving the performance especially for large-scale motors is limited by current through rotor and stator which causes temperature rise. So, effective cooling system for rotor and stator is required to achieve a better performance for large-scale motors.

A new design method for inlet guide vanes is developed to use for hydraulic bulb turbines. Guide vanes are fixed by conical surfaces with a common vertex. The thickness of blades is constant and they have cusped trailing edge and rounded leading edge. An analysis method was developed by $\mathrm{Wu}$ [5] for inviscid flow through a turbo machine. According to this method, the solution of the flow field can be obtained by iterative of two-dimensional flow which is defined on stream surfaces. This method can also be simplified using through flow analysis approach. In this simplification, all surfaces in S2 (meridional surface) become identical and S1 (blade-to-blade surface) is assumed to be generated from streamlines of the flow which are computed on S2 surfaces.
An axial fan was developed with hollow blades. It generates internal flow exiting as free jet. The internal flow influences the external one at the tip of the hollow blade in trailing edge region. Aerodynamic characteristic was measured for both fans with and without internal flow. From this analysis, turbulence intensities and circulation on the suction side of the hollow blade were dramatically decreased. In opposite, velocity presented higher amounts at the fan with internal flow.

In addition, by adding internal flow in the axial fan, the flow separation at the trailing edge region on the suction side at the tip of the hollow blade was minimized and pressure difference and efficiency had significant improving.

A guide vane system was designed with mini hydraulic turbine using quasi three- dimensional method which is combined with panel and streamline curvature methods for blade-to-blade and meridional flows, respectively.

Experimental results for pressure and velocity distributions obtained with five-hole probe in comparing with the numerical amounts obtained from FLUENT for viscous and inviscid flows. Experimental values showed a deviation from design ones in angular momentum near the inner casing, but these values were almost the same with a negligible approximation for angular momentum distribution at the exit of the guide vane system. Numerical results obtained from FLUENT code are validated base on the agreement with empirical results. The quasi three-dimensional method is also confirmed to be a suitable method for this type of applications.

\section{References}

1. Bleier F P (1998). Fan Handbook: selection, application, and design, McGraw-Hill, New York.

2. Borello D, Hanjalic Ket al. (2007). Computation of tip-leakage flow in a linear compressor cascade with a second-moment turbulence closure, International Journal of Heat and Fluid Flow, vol 28(4), 587-601.

3. Chang $\mathrm{C} \mathrm{C}$ et al., (2010). Air cooling for a large-scale motor, Applied Thermal Engineering, vol 30(11), 1360-1368.

4. Eberlinc M et al. (2009). Modification of axial fan flow by trailing edge self-induced blowing, Journal of Fluids Engineering, vol 131(11), 111104-1-111104-7.

5. Eberlinc M, B Širok et al. (2009). Experimental investigation of the interaction of two flows on the axial fan hollow blades by flow visualization and hot-wire anemometry, Experimental Thermal and Fluid Science, vol 33(5), 929-937.

6. Eberlinc M, Širok B et al. (2009). Patented hollow blades of the axial fan with trailing edge self-induced blowing, 
Benthem Science Publishers Recent Patents on Mechanical Engineering, vol 2(1), 1-7.

7. Eberlinc $M$ et al. (2007). Axial turbine machine hollow blade with internal and external flow field, The Slovenian Intellectual Property Office, Patent No. P. 200700271.

8. Eberlinc $M$ et al. (2008). Influence on the axial flow fan aerodynamic characteristic due to an introduction of internal secondary flow, ASME. Fluids Engineering Division Summer Conference, Jacksonville, Florida, USA.

9. Eberlinc $\mathrm{M}$ et al. (2009). Numerical and experimental investigation of axial fan with trailing edge self-induced blowing, Forschung im Ingenieurwesen, vol 73(3), 129-138.

10. Ferro L, Gato L et al. (2010). Design and experimental validation of the inlet guide vane system of a mini hydraulic bulb-turbine, Renewable Energy, vol 35(9), 1920-1928.

11. Garg V K (2002). Heat transfer research on gas turbine airfoils at NASA GRC, International Journal of Heat and Fluid Flow, vol 23(2), 109-136.

12. Lakshminarayana B (1996). Fluid dynamics and heat transfer of turbomachinery, Secondary Title, John Wiley, New York.

13. Liu S H, Huang R F (2010). Computational and experimental investigations of performance curve of an axial flow fan using downstream flow resistance method, Experimental Thermal and Fluid Science, vol 34(7), 827-837.

14. Meyer C j, and Kröger D G (2001). Numerical simulation of the flow field in the vicinity of an axial flow fan, International Journal for Numerical Methods in Fluids, vol 36(8), 947-969.
15. Murthy B et al. (2007). Hollow self-inducing impellers: Flow visualization and CFD simulation, Chemical Engineering Science, vol 62(14), 3839-3848.

16. Rhee S (2003). Analysis of a jet-controlled high-lift hydrofoil with a flap, Ocean Engineering, vol 30(16), 2117-2136.

17. Schlichting H, and K Gersten (2004). Boundary-layer theory, Springer.

18. Standard B (1997), Fans for general purpose. International Organization for Standardization: ISO 5801, Fans for General Purpose: Part 1: Performance Testing Using Standardized Airways.

19. Sutliff D L et al. (2002). Low-speed fan noise reduction with trailing edge blowing, International Journal of Aeroacoustics, vol 1(3), 275-305.

20. Wallis R (1983). Axial flow fans and ducts, Secondary Title, John Wiley \& Sons, New York.

21. Woodward R P, Fite E B (2007). Noise benefits of rotor trailing edge blowing for a model turbofan, 45th Aerospace Sciences Meeting and Exhibit, AIAA.

22. Yakhot A et al. (1989). Renormalization group formulation of large-eddy simulations, Journal of Scientific Computing, vol 4(2), 139-158.

23. Yakhot V et al. (1992). Development of turbulence models for shear flows by a double expansion technique, Physics of Fluids A: Fluid Dynamics, vol 4(7), 1510-1520.

24. Yakhot V, and Orszag S A (1986). Renormalization group analysis of turbulence I. Basic theory, Journal of Scientific Computing, vol 1(1), 3-51. 